Article

\title{
Antimicrobial Activity and the Effect of Green Tea Experimental Gels on Teeth Surfaces
}

\author{
Corina Voina ${ }^{1,+}$, Ada Delean ${ }^{1,+}$, Adriana Muresan ${ }^{2}$, Madalina Valeanu ${ }^{3}$, \\ Amalia Mazilu Moldovan ${ }^{4, *}$, Violeta Popescu ${ }^{4}$, Ioan Petean ${ }^{5}$, Razvan Ene ${ }^{6,7}$,
} Marioara Moldovan ${ }^{8}$ and Stanca Pandrea ${ }^{9}$

1 Department of Odontology and Oral Pathology, Faculty of Dental Medicine, University of Medicine and Pharmacy "Iuliu Hațieganu”, 33 Motilor Street, 400001 Cluj-Napoca, Romania; dr.corinaionescu@gmail.com (C.V.); adadelean@yahoo.com (A.D.)

2 Department of Functional Sciences, Faculty of Medicine, University of Medicine and Pharmacy "Iuliu Hatieganu", 3 Clinicilor Street, 400006 Cluj-Napoca, Romania; muresanadriana@yahoo.com

3 Department of Medical Informatics and Biostatistics, Faculty of Medicine, University of Medicine and Pharmacy “Iuliu Hatieganu', 6 Pasteur Louis Street, 400349 Cluj-Napoca, Romania; madalina.valeanu@gmail.com

4 Physics and Chemistry Department, Technical University of Cluj-Napoca, 28 Memorandumului Street, 400114 Cluj-Napoca, Romania; violetap2003@gmail.com

5 Faculty of Chemistry and Chemical Engineering, Babes Bolyai University, 11 Arany Janos Street, 400028 Cluj-Napoca, Romania; petean.ioan@gmail.com

6 University of Medicine and Pharmacy Carol Davila Bucharest, 050474 Bucharest, Romania; razvan77ene@yahoo.com

7 Orthopedics and Traumatology Department, Bucharest Emergency University Hospital, 050098 Bucharest, Romania

8 Institute of Chemistry Raluca Ripan, Babes-Bolyai University, 30 Fantanele Str, 400294 Cluj-Napoca, Romania; mmarioara2004@yahoo.com

9 Department of Microbiology, Faculty of Medicine, University of Medicine and Pharmacy "Iuliu Hatieganu", 6 Pasteur Louis Street, 400349, Cluj-Napoca, Romania; stanca_lucia_pandrea@yahoo.com

* Correspondence: amalia.mazilu@gmail.com

+ These authors contributed equally to this work.

Received: 14 May 2020; Accepted: 29 May 2020; Published: 31 May 2020

\begin{abstract}
Among esthetic procedures, teeth whitening is a common and often used treatment for patients who seek good teeth appearance. We developed an experimental green tea extract and an experimental green tea gel for enamel restoring treatment after bleaching. We also tested the antibacterial and antifungal effect of the experimental extract against specific endodontic and cariogenic microorganisms. The green tea extract antibacterial action was determined by the disk-diffusion method using Peptostreptococcus anaerobius (ATCC27337), Corynebacterium xerosis (ATCC 373), Streptococcus mutans (ATCC 25175) and Candida albicans (ATCC 10231) strains. Enamel microstructure was investigated by SEM analysis, and surface details were revealed by AFM. The inhibition zones around the wells showed evident antimicrobial activity of the experimental extract. In the presence of Candida albicans (ATCC 10231), the extract showed no antifungal activity. The enamel's surface roughness and hydroxyapatite prism aspects were the parameters followed throughout the study. The experimental green tea extract is efficient against some microorganisms commonly found in the oral cavity. However, the studied extract had no antifungal effect. The results show that after bleaching with the experimental gel, we obtained the best surface parameters, similar to healthy enamel.
\end{abstract}

Keywords: SEM; AFM; remineralization; green tea; antimicrobial; disk-diffusion; surface roughness 


\section{Introduction}

Green tea is the most consumed beverage, after water [1], and it is obtained by infusing in hot water the dried leaves of Camellia sinensis, a plant belonging to the Theaceae family. This plant is evergreen, and it is cultivated in tropical and subtropical climates, especially in China, Sri Lanka and Japan [2].

Previous studies in the literature have associated green tea with multiple therapeutic effects. It has a strong antioxidant action due to its catechin content and also contains condensed and hydrolysable tannins, which are also responsible for some therapeutic effects [3].

It was demonstrated that EGCG (epi-gallo-catechin-gallate) has the role of inhibiting the growth of microorganisms responsible for inflammation and bone resorption, according to in vitro studies on green tea catechins, regarding periodontal disease [4]. It has also been suggested that green tea catechins (EGCG) offer a promising prospect for the development of a new adjuvant treatment for chronic marginal periodontitis due to their ability to decrease the secretion of MMP (matrix-metalloproteinase), which are important tissue-destroying enzymes produced by mucous membranes and immune cells [5].

In endodontics, an herbal irrigation solution is needed because of the side effects, especially the toxicity, of the most used irrigants. Green tea has shown in some in vitro studies an antibacterial effect on the biofilm Enterococcus faecalis, which is the most common species isolated from the root canal of teeth with refractory and recurrent apical periodontitis [6-8].

Nowadays, the concerns of dentists and patients have increasingly focused on improving the aesthetic appearance of teeth. Procedures commonly used in dental clinics include tooth whitening. This is a relatively safe clinical procedure, well received by patients, but not without undesirable side effects.

The products used for bleaching have carbamide peroxide as their main active ingredient. In contact with oral fluids, it decomposes into water, urea and oxygen, which by oxidation exerts the whitening effect. Dental sensitivity is one of the most common side effects due to the action of oxygen free radicals [9] resulting from the decomposition of carbamide peroxide [10]. Some studies explain this by the ability of peroxide to diffuse through dentinal tubes to the pulp chamber [11].

Additionally, Gonzales et al. demonstrated, in their histological research, the existence of slight inflammatory modification into the pulp tissue [12].

Another adverse effect of the tooth bleaching procedure is the morphological alterations of the enamel [13]. Other studies have shown changes in tooth enamel after whitening due to the loss of calcium ions from its structure [14].

The research in this study aimed to prepare and characterize a new product for the protection of dental hard tissues after the tooth bleaching procedure. This preparation is represented by the natural extract from green tea leaves (Camellia sinensis). The antibacterial studies proposed in this study and performed in vitro test the antimicrobial efficacy of the experimental extract against the main microorganisms responsible for the production of dental caries and other endodontic or periodontal diseases. Additionally, we followed in this study the effect of the experimental gel on the surface roughness of tooth enamel following the teeth whitening process, which is known from the literature as a procedure extremely requested by patients, but with undesirable side effects, in mainly surface lesions of the enamel, which over time can promote or even trigger carious lesions.

The objectives of this study were to test the antimicrobial and antifungal action of an experimental extract based on Camellia sinensis on bacterial and fungal strains commonly identified on hard dental tissues, as well as to evaluate the effect of the experimental extract on surface roughness of bleached enamel with carbamide peroxide based gels. 


\section{Materials and Methods}

\subsection{Materials}

Twenty-eight healthy third molars, extracted for orthodontic purposes, were taken in the present study and randomly divided into four experimental groups as follows: one group of healthy, untreated teeth, which served as a control group, a group of stained but not bleached teeth, which served as a stained control group and two groups of teeth designated as experimental groups. For this study we received approval from the Ethics Commission of the University of Medicine and Pharmacy Cluj-Napoca, under approval number 180/06.05.2020.

We also used for this study Opalescence $16 \%$, a carbamide peroxide (PC) whitening gel provided from Ultradent Products (South Jordan, UT, USA), and we prepared and used experimental green tea extract and experimental bleaching gel.

The present study was performed on 4 microbiological strains from ATCC (American Type Culture Collection) accredited cultures, purchased from Microbiologics, St. Cloud, Minnesota, USA. These are Streptococcus mutans (ATCC 251175), Peptostreptococcus anaerobius (ATCC 27337), Corynebacterium xerosis (ATCC 373) and Candida albicans (ATCC 10231).

\subsection{Preparing the Experimental Extract of Camellia Sinensis and the Experimental Bleaching Gel}

The green tea extract was developed within the "Raluca Ripan" Chemistry Research Institute of the Babes-Bolyai University of Cluj-Napoca. Four experimental extracts of different concentrations were made with different infusion times of the plant as follows: in three batches of $100 \mathrm{~mL}$ of boiled distilled water were added $35 \mathrm{~g}, 40 \mathrm{~g}$ and $50 \mathrm{~g}$ green tea leaves (R. Twining and Company Limited, London, UK).

The 35\% extract solution was left to infuse for $15 \mathrm{~min}$, the $40 \%$ solution for $30 \mathrm{~min}$, and the $50 \%$ extract was infused for $60 \mathrm{~min}$. The fourth extract was prepared as follows: $20 \mathrm{~g}$ of green tea leaves were added to $200 \mathrm{~mL}$ of boiled distilled water and infused for $10 \mathrm{~min}$. Subsequently, the products were filtered to remove possible impurities and stored in sterile containers at $4{ }^{\circ} \mathrm{C}$ for later use. To obtain the experimental bleaching gel, $16 \%$ carbamide peroxide was added to the extract solution.

\subsection{Growth of the Studied Bacterial and Fungal Strains}

To obtain the study cultures, strains from ATCC (American Type Culture Collection) accredited cultures were cultured on specific culture media. Thus, Corynebacterium xerosis (ATCC 373) was inoculated on the culture medium tryptic soy agar with $5 \%$ sheep blood (Biomerieux, Lyon, France) for $48 \mathrm{~h}$ at a temperature of $35 \pm 1{ }^{\circ} \mathrm{C}$ in an atmosphere of $5 \% \mathrm{CO}_{2}$, after which the colonies were replicated on the same media and in the same conditions $\left(48 \mathrm{~h}, 35 \pm 1{ }^{\circ} \mathrm{C}, 5 \% \mathrm{CO}_{2}\right)$ to obtain the working culture. Special $\mathrm{CO}_{2}$-generating bags (GenBag $\mathrm{CO}_{2}$, Biomerieux, Lyon, France) were used to obtain the specific growth atmosphere.

Streptococcus mutans (ATCC 25175) was inoculated on agar with $5 \%$ sheep blood media culture (Biomerieux, Lyon, France) and incubated for $48 \mathrm{~h}$ at $35 \pm 1{ }^{\circ} \mathrm{C}$ in the atmosphere $5 \% \mathrm{CO}_{2}(\mathrm{GenBag}$ $\mathrm{CO}_{2}$, Biomerieux, Lyon, France). The culture was replicated on the same culture media and in the same conditions, obtaining the working culture after another $48 \mathrm{~h}$.

Peptostreptococcus anaerobius (ATCC 27337) was inoculated on anaerobic blood agar (Scaedler and Brucella Blood Agar), Biomerieux, Lyon, France, then incubated at $35^{\circ} \mathrm{C}$ in an anaerobic atmosphere for $48 \mathrm{~h}$ (GenBag anaerobic, Biomerieux, Lyon, France). It was then replicated on the same medium and in the same conditions, and the working culture was obtained after another $48 \mathrm{~h}$.

Candida albicans (ATCC 10231) was cultivated on Sabourand agar medium (Biomerieux, Lyon, France) for $48 \mathrm{~h}$ at $37^{\circ} \mathrm{C}$. The culture was replicated and cultivated under the same conditions, obtaining the working culture. 


\subsection{Preparation of Dental Specimens}

The 28 molars under study were mounted in self-curing acrylate prisms (Duracryl Plus, Spofadental Inc, Jičín, Czech Republic) up to the enamel-dentin junction level, keeping the entire coronary portion free.

\subsection{Staining Protocol}

The two experimental groups and the control stained group were subjected to a staining process, the molars being immersed in coffee solution $8 \mathrm{~h} /$ day, for 3 consecutive days. The coffee solution was prepared in the Saeco Pico Baristo machine in espresso mode using $5 \mathrm{~g}$ of Lavazza coffee (Luigi Lavazza SpA, Torino, Italy) per $100 \mathrm{~mL}$ of water. A freshly brewed coffee solution was used at each staining session. Between staining sessions, the teeth were kept immersed in artificial saliva at $37^{\circ} \mathrm{C}$.

\subsection{Bleaching Protocol}

The bleaching protocol aimed to simulate a whitening treatment at home using individualized trays. Thus, 16\% carbamide peroxide whitening gel (Opalescence 16\%, Ultradent Products, South Jordan, UT, USA) was applied to the Opalescence gel group for $6 \mathrm{~h} /$ day, for 7 consecutive days. The experimental gel study group underwent experimental bleaching gel for $6 \mathrm{~h} /$ day for 7 consecutive days. At the end of each bleaching session, the teeth were washed for $60 \mathrm{~s}$ under running water and then stored in artificial saliva at $37^{\circ} \mathrm{C}$.

At the end of the experiment, slices from the vestibular part of the molars from each study group were taken in order to analyze the appearance of the enamel by scanning electron microscopy and atomic force microscopy.

\subsection{Determination of Antibacterial and Antifungal Activity}

To determine the antibacterial activity of the experimental solutions, we used the standard procedure recommended by EUCAST, meaning the diffusimetric method (Eucast Disk Diffusion Method for Antimicrobial Susceptibility Testing, version 8.0, January 2018).

Working cultures of Corynebacterium xerosis (ATCC 373) and Streptococcus mutans (ATCC 25175) were inoculated on Mueller-Hinton Agar medium with 5\% defibrinated horse blood (Biomerieux, Lyon, France) and $20 \mathrm{mg} / \mathrm{L} \beta-\mathrm{NAD}$ (Mueller-Hinton F, Biomerieux, Lyon, France). The two bacterial cultures were incubated at a temperature of $35 \pm 1{ }^{\circ} \mathrm{C}$ for $18 \pm 2 \mathrm{~h}$ in an atmosphere of $5 \% \mathrm{CO}_{2}(\mathrm{GenBag}$ $\mathrm{CO}_{2}$, Biomerieux, Lyon, France).

The culture of Peptostreptococcus anaerobius (ATCC 27337) was inoculated on brucella blood agar culture media (Biomerieux, Lyon, France) and incubated at $35^{\circ} \mathrm{C}$ in an anaerobic atmosphere (anaerobic GenBag, Biomeriex, Lyon, France) for $48 \mathrm{~h}$.

The culture of Candida albicans (ATCC 10231) was grown on Sabouraud medium (Biomerieux, Lyon, France) and $0.5 \mathrm{~mL}$ McFarland inoculum and incubated at $30^{\circ} \mathrm{C}$ for $72 \mathrm{~h}$.

After obtaining the test cultures, we impregnated sterile antibiogram discs (Oxoid, Hamptshire, UK) with different concentrations of green tea extract or we created sterile wells with a diameter of $6 \mathrm{~mm}$ on the culture media plate, reading the diameters of the inhibition zones. Clear areas of inhibition around each disc/well revealed the presence of antimicrobial activity for each solution used. For each concentration and amount inoculated, 7 repeated determinations were performed. In order to determine the antimicrobial effect of the experimental extract, according to the CLSI (Clinical and Laboratory Standards Institute) standards, it was necessary to determine the standard reference values of the inhibition zones for each bacterial and fungal strain.

For this, we used impregnated discs with standard antimicrobial substances, according to CLSI, from Bio-Rad, Hercules, CA, USA, as follows: for Corynebacterium xerosis (ATCC 373) and Streptococcus mutans (ATCC 25175) we used benzylpenicillin 1 unit, clindamycin $2 \mu \mathrm{g}$, vancomycin $5 \mu \mathrm{g}$, gentamicin $10 \mu \mathrm{g}$ and ampicillin $2 \mu \mathrm{g}$. Streptococcus pneumoniae (ATCC 49619) strain purchased 
from Microbiologics, St. Cloud, Mn, USA, was used for internal quality control. The control strain was inoculated onto Mueller-Hintor Agar culture media with 5\% defibrinated horse blood (Biomerieux, Lyon, France) and $20 \mu \mathrm{g} / \mathrm{L} \beta$-NAD (Mueller-Hinton F, Biomerieux, Lyon, France). After obtaining the working cultures, the discs impregnated with antibiotic specific for each microbial strain were placed in plates, reading the diameters of the inhibition zones after $48 \mathrm{~h}$. Although not standardized for anaerobes, we also used the diffusimetric method to test Peptostreptococcus anaerobius (ATCC 27337) grown on brucella blood agar (Biomerieux, Lyon, France) and incubated in an anaerobic atmosphere (GenBag anaerobic, Biomerieux, Lyon, France).

\subsection{SEM and AFM Analysis of Tooth Specimens}

All study groups were evaluated by scanning electron microscopy (SEM) to highlight the change in tooth surface as a result of staining and then whitening treatment. For comparison, we used as a control the group of teeth not subjected to the processes of staining and bleaching. The samples were examined using an Inspect-S microscope (FEI Company, Hillsboro, OR, USA). Several image enlargement units were used, and the recordings were made on the surface of the tooth enamel. Atomic force microscopy (AFM) was performed using a JEOL JSPM 4210 scanning probe microscope, Japan. The topographic images were recorded in tapping mode using NSC 15 cantilevers produced by Micromasch Co. Bulgaria (resonant frequency $325 \mathrm{kHz}$ and force constant $40 \mathrm{~N} / \mathrm{m}$ ). The enamel microstructure was scanned at an area of $5 \mu \mathrm{m} \times 5 \mu \mathrm{m}$ at a scan rate of about $1 \mathrm{~Hz}$, and the nanostructure was scanned at area of $1 \mu \mathrm{m} \times 1 \mu \mathrm{m}$ at a rate of $1.5-2 \mathrm{~Hz}$. The AFM topographic images were processed using WinSPM 2.0 Processing soft powered by JEOL for the AFM microscopes. For each slice of tooth examined, 7 repeated determinations were performed.

\subsection{Statistical and Mathematical Methods}

The SPSS software package version 21.0 (SPSS Inc., Chicago, IL, USA) was used for statistical analysis and graphical representations. The acceptable error threshold was $\alpha=0.05$. To describe continuous quantitative data, we used arithmetic mean, standard deviation (SD), minimum, maximum and standard error. The Kruskal-Wallis ANOVA test was used to test the differences between the independent groups investigated. The Mann-Whitney test was used in the post-hoc analysis when significant differences were identified by the Kruskal-Wallis ANOVA test. The Spearmann correlation coefficient was calculated to determine the links between quantitative variables and a non-normal distribution within the groups. Colton's rules were used to interpret the correlation coefficient.

\section{Results}

The experimental extract of Camellia sinensis developed in the present study was evaluated from a microbiological point of view by testing the antimicrobial activity on different microbial strains frequently identified in the oral cavity. The diffusimetric method is based on the property of antimicrobial substances to diffuse in a solid culture media, on which the bacterial culture to be tested is cultivated. The interpretation was made according to the diameter of the inhibition zone, measured with a ruler, including the diameter of the well/disc. The values obtained in millimeters assessed the strain as sensitive, intermediately sensitive or resistant to the test substance (Figures 1-3).

It was necessary to relate the values recorded to the standard reference values of the inhibition zones for each tested microbiological strain in order to determine the antimicrobial effect of the experimental extract, according to CLSI (Clinical and Laboratory Standards Institute) norms. These are shown in Table 1. 

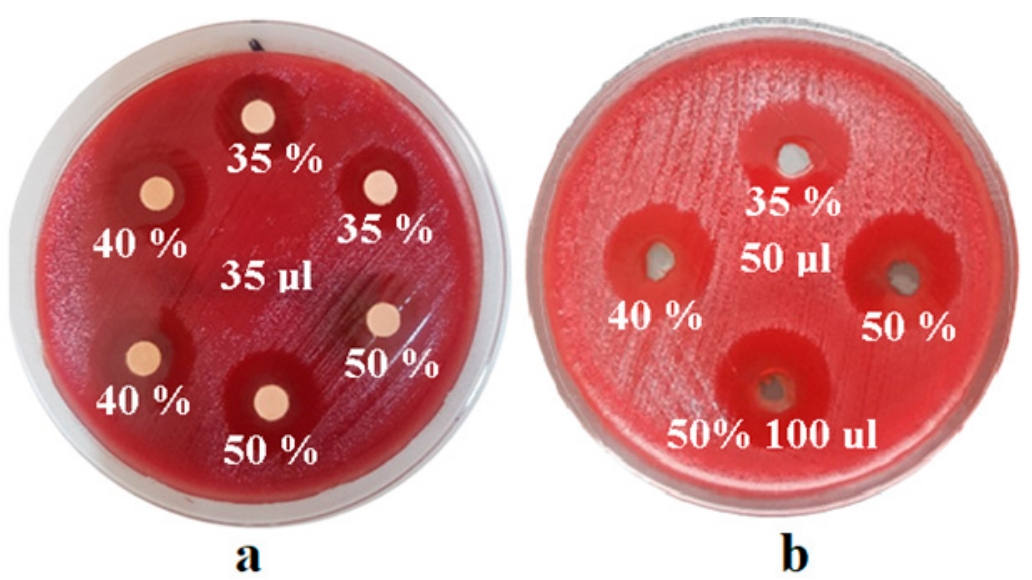

Figure 1. Inhibition diameters of Peptostreptococcus anerobius culture on inoculation of $35 \mu 1$ of $35 \%$, $40 \%$ and $50 \%$ extract (a) and diameters of inhibition zones of Peptostreptococcus anaerobius culture on inoculation of $50 \mu \mathrm{l}$ of $35 \%, 40 \%$ and $50 \%$ extract, as well as $100 \mu \mathrm{l}$ extract $50 \%$ (b).

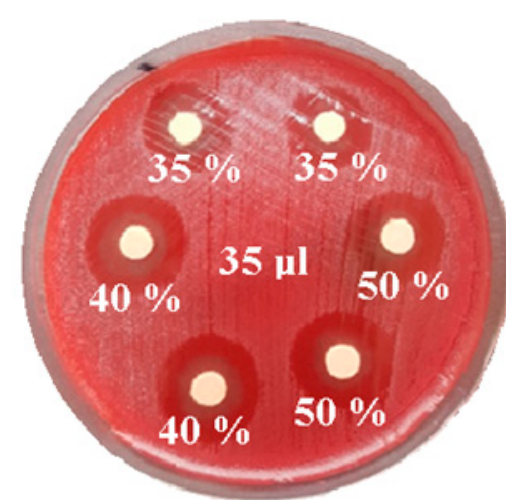

a

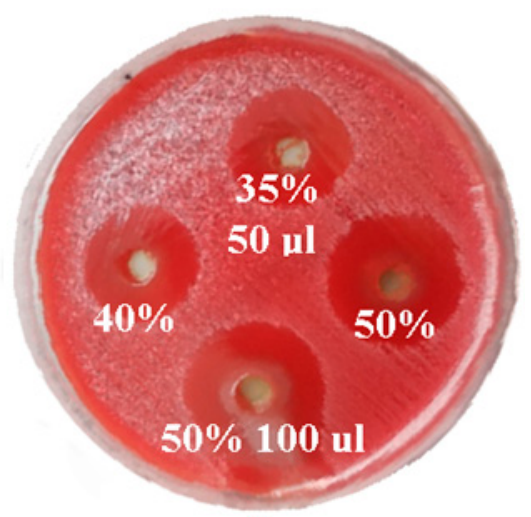

b

Figure 2. Corynebacterium xerosis culture inhibition diameters when inoculating $35 \mu \mathrm{l}$ of $35 \%, 40 \%$ and $50 \%$ extract (a) and Corynebacterium xerosis culture inhibition diameters when inoculating $50 \mu 1$ of $35 \%$, $40 \%$ and $50 \%$ extract, as well as $100 \mu$ l extract $50 \%$ (b).

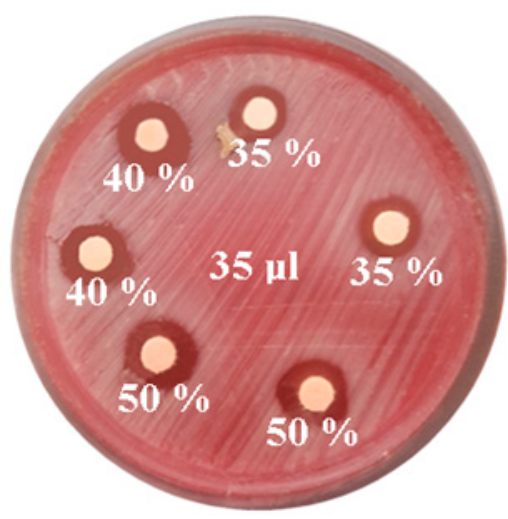

$\mathbf{a}$

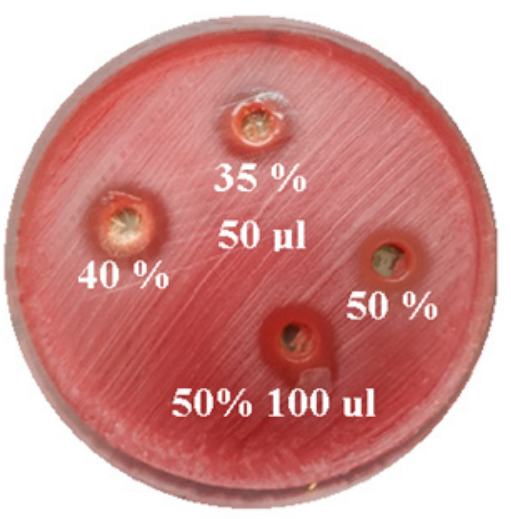

b

Figure 3. Streptococcus mutans culture inhibition diameters when inoculating $35 \mu \mathrm{l}$ of $35 \%, 40 \%$ and $50 \%$ extract (a) and Streptococcus mutans culture inhibition diameters when inoculating $50 \mu 1$ of $35 \%$, $40 \%$ and $50 \%$ extract, as well as $100 \mu$ l extract $50 \%$ (b). 
Table 1. Mean values of diameters of zones of inhibition in $\mathrm{mm}$ for CLSI reference substances.

\begin{tabular}{lcccc}
\hline & \multicolumn{4}{c}{ Diameter of the Inhibition Zones } \\
& C.xerosis & S. mutans & S. pneumoniae & C. albicans \\
\hline Benzylpenicilin $1 \mathrm{u}$ & 32 & 22 & & \\
Ampicilin $2 \mu \mathrm{g}$ & & 24 & & \\
Clyndamicin $2 \mu \mathrm{g}$ & 28 & 20 & 22 & \\
Vancomycin $5 \mu \mathrm{g}$ & 20 & 20 & 18 & \\
Gentamicin $10 \mu \mathrm{g}$ & 24 & & & \\
Oxacillin $1 \mu \mathrm{gg}$ (only & & & 22 & \\
for S.pneumoniae) & & & & \\
Fluconazole $10 \mu \mathrm{g}$ & & & & \\
\hline
\end{tabular}

The mean values of the zones of inhibition, after measuring the inhibition diameters in $\mathrm{mm}$ for the strain Peptostreptococcus anaerobius (ATCC 25337) and for the strain Corynebacterium xerosis (ATCC 373), are illustrated in Figure 4.

The mean values of the inhibition zones, after measuring the inhibition diameters in $\mathrm{mm}$ for the S. mutans (ATCC 25175) strain, are illustrated in Figure 5.

On the Candida albicans (ATCC 10231) strain, the experimental extracts did not exert any antifungal effect, as illustrated in Figure 6.

Figure 7 shows the microstructural aspects specific to the untreated healthy enamel. Its aspect was characterized by a relatively smooth surface that showed signs of tooth wear due to the abrasive effect of mastication correlated with the acidity of food (Figure 7a). These were manifested by the erosion and dislocation of HAP (hydroxyapatite) crystallites inside the prisms that became visible in the form of a series of fine points.

At a medium magnification, as seen in Figure $7 \mathrm{~b}$, we noticed that in the eroded areas the prisms became very visible, having a circular depression aspect leading to a honeycomb type microstructure. This correlated with data from the literature showing that acid demineralization of prisms occurs with the dislocation of nanostructure HAP crystallites inside them, forming a honeycomb structure. The honeycomb-shaped portions were clearly distinguished from the unaffected enamel portions. Their morphology was more obvious at large magnification, as seen in Figure $7 c$, where in the central part of the image we see the perfect enamel area, and towards the left and top left of the image appear the demineralized portions.

The overall appearance of the stained enamel with coffee solution is shown in Figure 8a. We noticed that the coffee residues entered the depressions of the prisms caused by chewing and filling them. By this, we would expect a uniformity of the enamel surface but the detail at medium magnification, as seen in Figure 8b, showed that there were crusts of thicker deposits that made the relief of the enamel significantly uneven. Alteration of the prisms was more obvious at high magnification, as seen in Figure 8c. The upper corner of the image reveals partially altered prisms and the central part of the image shows completely destructed enamel. The microstructural detail, as seen in Figure $8 c$, highlights such a deposition crust in which we observed advanced degradation of the enamel.

SEM analysis of the stained and bleached enamel with Opalescence gel revealed the smooth appearance of the enamel with small irregularities. We noticed a net change in appearance, highlighting the action of the bleaching gel by removing the chromophore deposits and traces of wear (Figure 9) by comparison with the untreated group. The whitening produced by Opalescence gel removed both the deposition crusts and the deposits penetrating the depressions in the prisms. Thus, the overall appearance of the enamel, as seen in Figure 9a, was much smoother and of a higher quality than the untreated healthy enamel. However, looking in more detail, as seen in Figure 9b,c, we observed significant morphological alterations in the enamel surface consistent with the data in the literature $[15,16]$. 


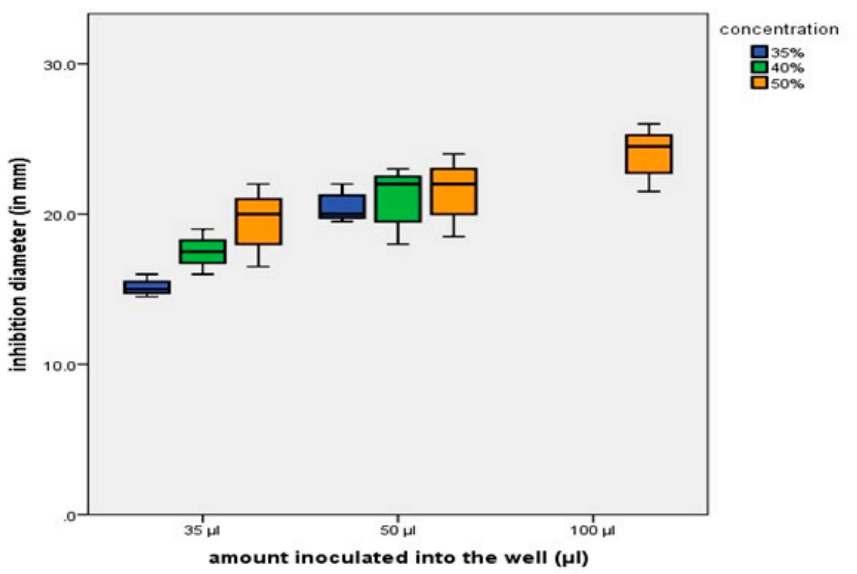

(a)

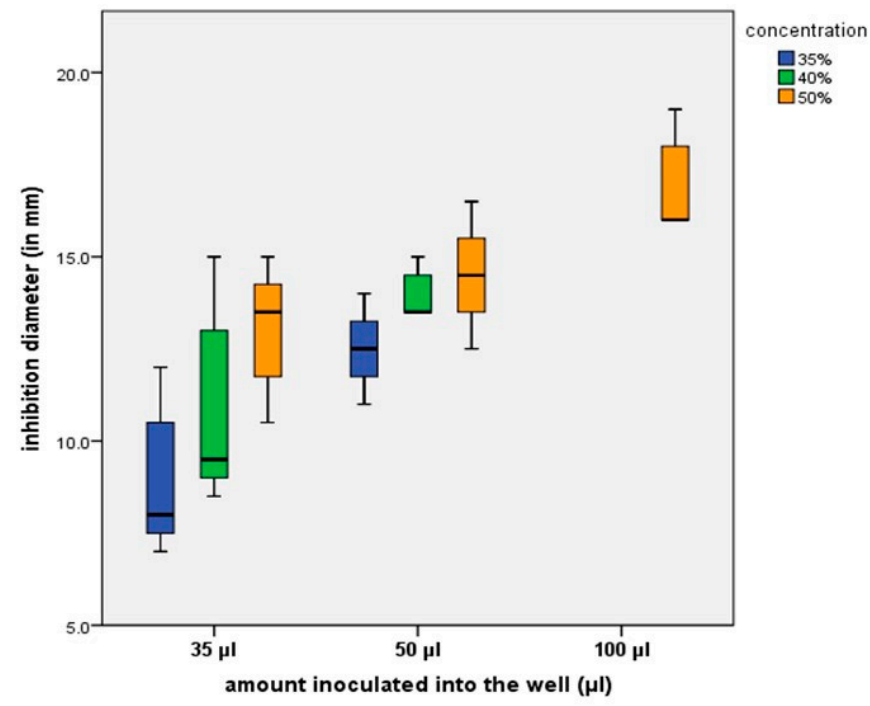

(b)

Figure 4. Mean values of inhibition diameters for P. anaerobius strain (a) and for C. xerosis strain (b) as a function of concentrations and quantities.

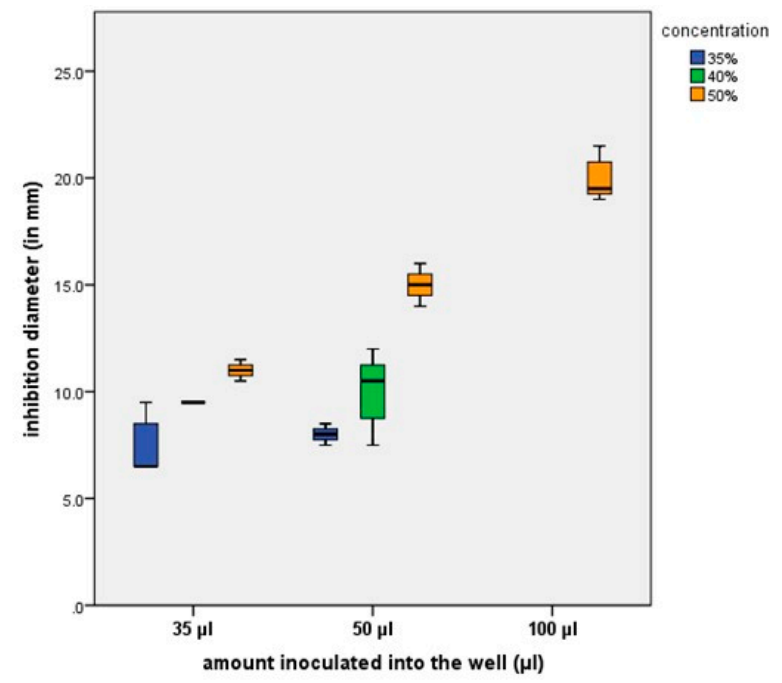

Figure 5. Mean values of inhibition diameters for $S$. mutans strain as a function of concentrations and quantities. 


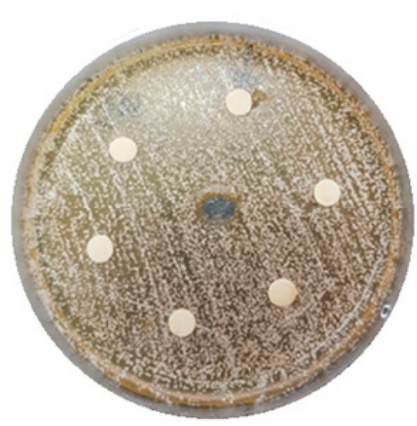

a

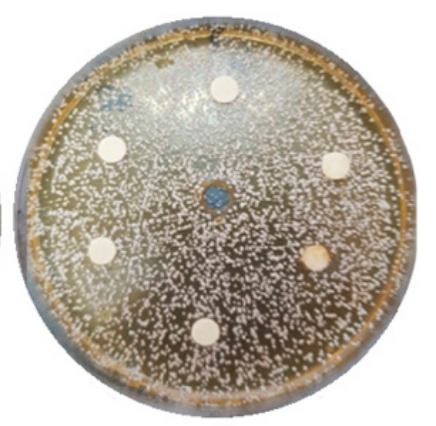

b

Figure 6. Appearance of Candida albicans culture plates $48 \mathrm{~h}$ after inoculation of experimental extract: inhibition diameters when inoculating $35 \mu \mathrm{l}$ of $35 \%, 40 \%$ and $50 \%$ extract (a) and inhibition diameters when inoculating $50 \mu \mathrm{l}$ of $35 \%, 40 \%$ and $50 \%$ extract, as well as $100 \mu \mathrm{l}$ extract $50 \%$ (b).

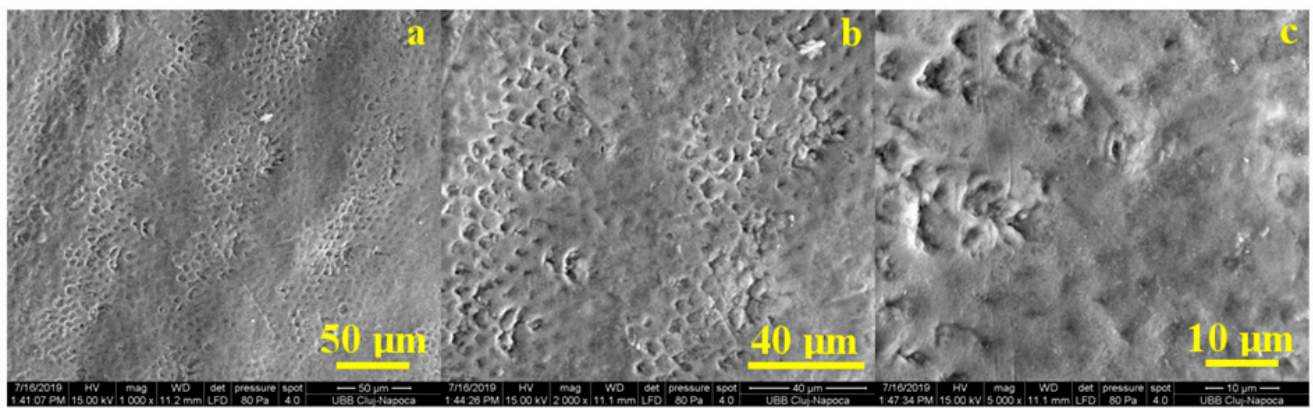

Figure 7. SEM aspect of untreated healthy enamel: (a) small magnification, (b) medium magnification, and (c) high magnification.

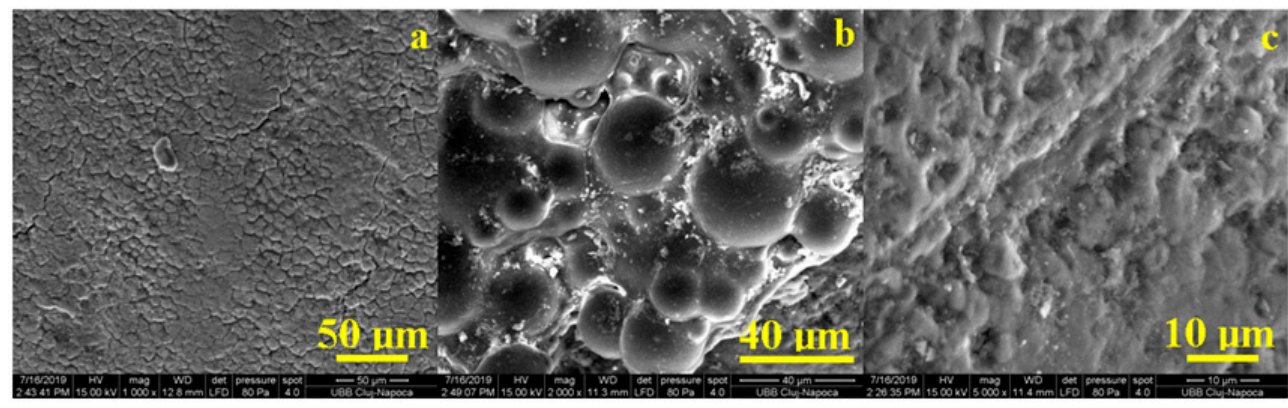

Figure 8. SEM aspect of stained enamel: (a) small magnification, (b) medium magnification, and (c) high magnification.

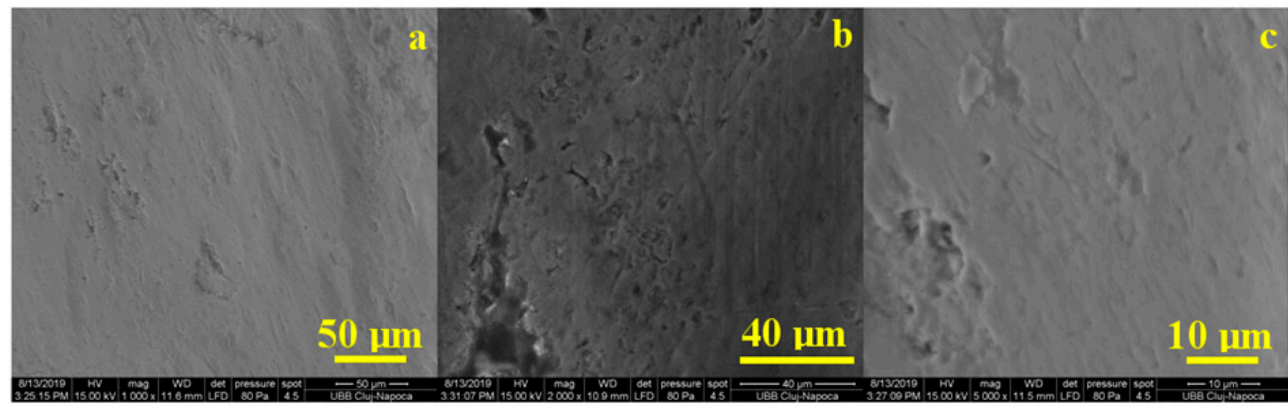

Figure 9. SEM appearance of bleached enamel with Opalescence gel: (a) small magnification, (b) medium magnification, and (c) high magnification. 
SEM analysis of the stained and bleached group with experimental bleaching gel led to the images in Figure 10. Both overall and in detail, there was an incredibly good removal of chromophore deposits and a restoration of enamel quality, very close to the perfect areas observed in untreated healthy enamel. The details in Figure 10b,c show how the experimental gel led to a very good restoration of the demineralized areas, completely filling the depressions in the prisms. A nanostructural analysis performed with atomic force microscope (AFM) is needed to highlight the restructuring of the HAP crystallite architecture.

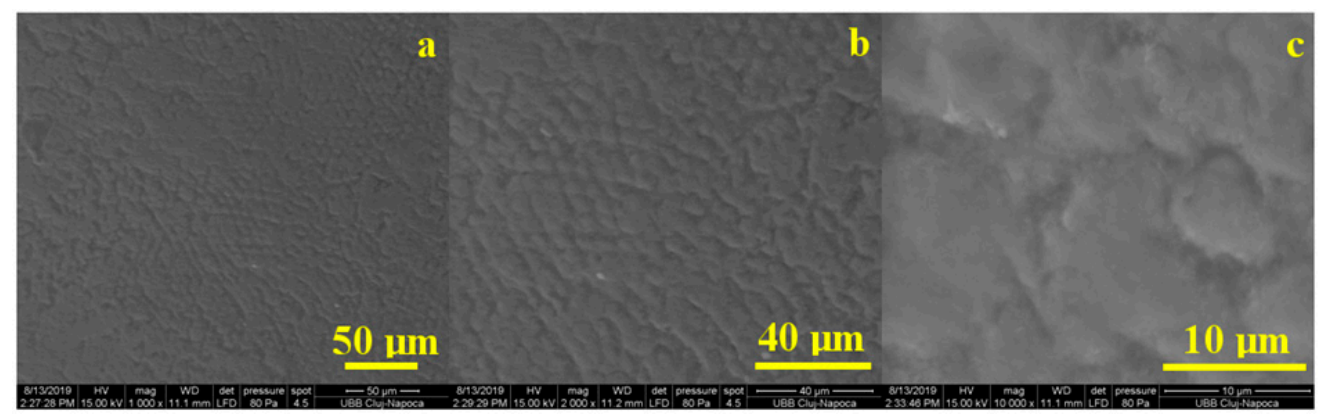

Figure 10. SEM appearance of stained and bleached enamel with experimental gel: (a) small magnification, (b) medium magnification, and (c) high magnification.

The HAP prisms of enamel had diameters between 5 and $7 \mu \mathrm{m}$, as shown from SEM imaging. The enamel surface was investigated by atomic force microscopy at a scanning area with a side of $5 \mu \mathrm{m}$ in order to observe their fine microstructure.

The healthy, untreated enamel, as seen in Figure 11a, captured in the central part of the image the appearance of the perfect enamel area, where the HAP crystallites were very well welded together. In the upper right corner of the image we noticed the edge of a depression sign that this portion corresponded to the edge of a partially demineralized prism, in line with those observed by SEM. Overall the surface was smooth and compact, resulting in a low roughness (Table 2).

The effect of enamel staining with coffee solution was dramatic at the fine microstructural level, as seen in Figure 11b, highlighting massive deposits that had a deep uneven effect that caused a significant increase in roughness and the disappearance of the enamel-specific structure. This shows that it was completely covered with coffee grounds.

After the bleaching process with Opalescence gel, the results showed a visually impressive enamel surface, confirmed by the quality of the AFM image in Figure 11c. The appearance of the surface was smooth and quite compact. The crystals observed on the surface were much larger and duller than those typical for healthy enamel. Probably, the Opalescence gel is very efficient in removing stains and chromophore residues and also acts on HAP crystallites, causing a relative chemical erosion of them. Thus, the roughness decreased significantly, but did not reach the typical value for healthy enamel.

The treatment with the experimental gel led to an enamel surface very close to that of the healthy one, as seen in Figure $11 \mathrm{~d}$. The appearance of the enamel after this treatment was compact with HAP crystallites very well welded together. The roughness decreased to a value close to that of healthy enamel after treatment (Table 2). This highlights a much gentler and more efficient cleaning of the enamel by the action of experimental gel.

The evolution of the appearance and diameter of HAP crystallites was studied with the AFM microscope at a scanning area with a side of $1 \mu \mathrm{m}$. The healthy enamel, as seen in Figure 12a, showed a compact structure of HAP nano-crystallites with a rounded shape and a diameter of $40 \mathrm{~nm}$ very well welded together. After staining with coffee, a degradation of the enamel nanostructure was observed, as seen in Figure 12b, which was manifested by the appearance of deposits and by the relative alteration of the crystallite size, increasing from 40 to $65 \mathrm{~nm}$. This is normal, if we take into consideration the deposits that have adhered to the surface, causing an effective doubling of the surface roughness. 
After the treatment with Opalescence gel, we noticed that the enamel surface became very clean and free of impurities, but the morphology of HAP crystallites remained significantly altered, such that the nanoparticles had an eroded, blunt appearance, with diameters around $80 \mathrm{~nm}$. Therefore, the major change induced by Opalescence occurred at the nanostructural level. Of course, this change had a very aesthetic overall appearance of bleached teeth, but the durability over time of the newly formed structure remains debatable, especially due to the remaining roughness, which was $14.7 \mathrm{~nm}$. The edges of the nanocrystallites, as seen in Figure 12c, could very easily become support for future deposits.

The experimental gel treatment also worked optimally at the nanostructural level, as seen in Figure $12 \mathrm{~d}$. We noticed a compact and smooth surface, very similar to the healthy enamel after the treatment. The transformation of the situation in Figure $12 \mathrm{~b}$ into that of Figure $12 \mathrm{~d}$ took place with a gentle removal of impurities, as well as facilitating the local remineralization of the enamel. The diameter of the HAP crystallites after treatment with the experimental gel was very close to the diameter of HAP prisms of healthy enamel, and the value of roughness at the nano level was very close.

Summarizing the microstructural and nanostructural aspects, we can conclude that the Opalescence gel had a strong aesthetic effect that involved quite severe changes in the enamel, including morphological alterations, and the experimental gel gave the enamel a typical aesthetic appearance for a clean and well maintained natural tooth involving a gentle cleaning mechanism that protects the mineral structure of the enamel starting from the nanostructural level.
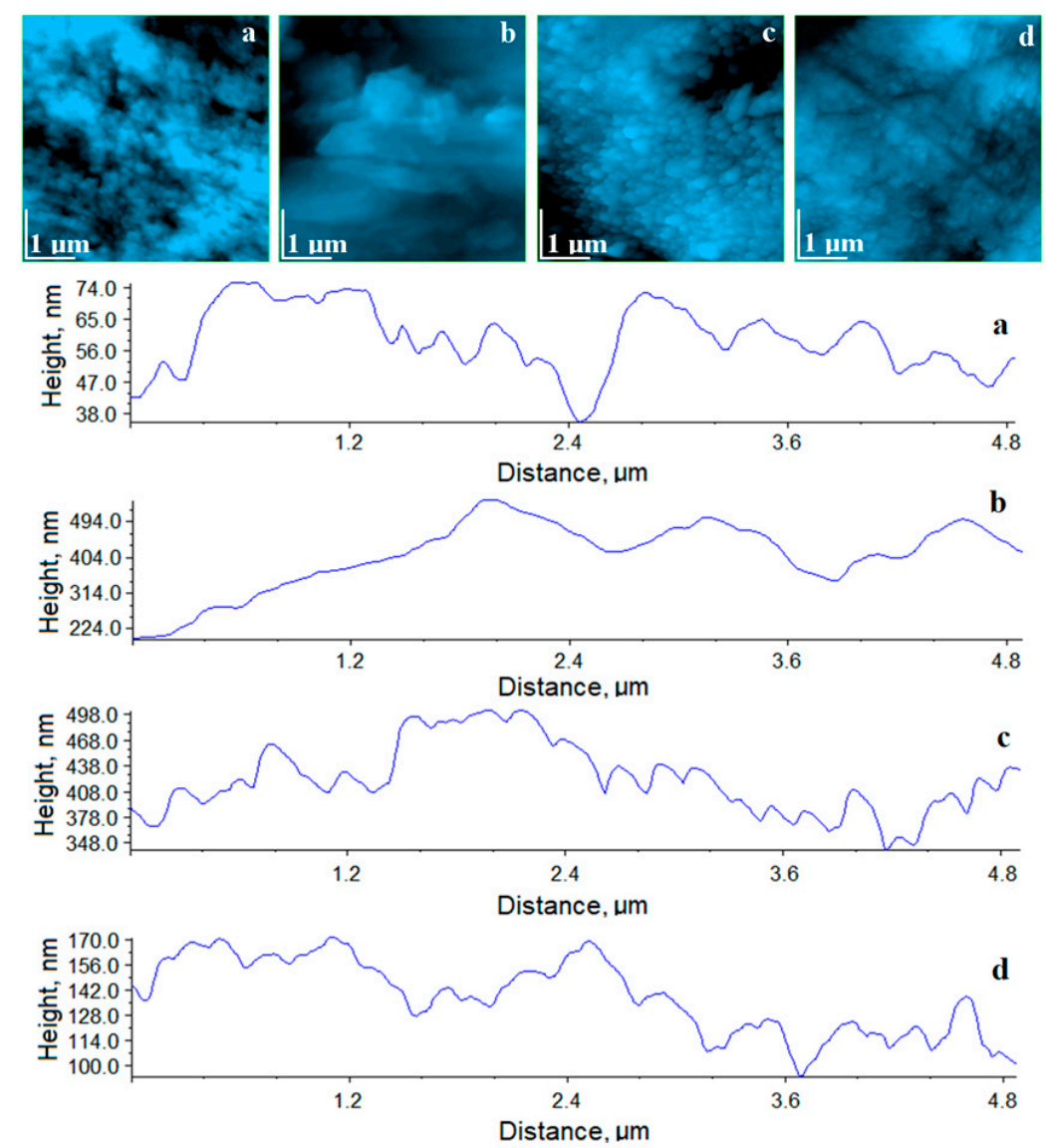

Figure 11. AFM topographic images of the fine microstructure of the samples: (a) healthy, untreated enamel, (b) stained enamel with coffee, (c) stained enamel and then treated with Opalescence gel, and (d) stained enamel and then treated with experimental bleaching gel. Two-dimensional profiles on the horizontal median of the topographic images are given. They are related to the roughness at the fine microstructure level, "Ra micro" in Table 2. 
Table 2. Enamel surface parameters measured with AFM.

\begin{tabular}{|c|c|c|c|c|c|}
\hline \multicolumn{2}{|c|}{ Parameter } & \multicolumn{4}{|c|}{ Enamel Sample } \\
\hline & & Healthy & Coffee stained & Opalescence gel & Experimental gel \\
\hline \multirow{5}{*}{ Ra micro., nm } & Minimum & 8.98 & 28.31 & 35.76 & 16.17 \\
\hline & Maximum & 22.8 & 35.42 & 77.21 & 49.75 \\
\hline & Arithm. mean & 14.23 & 31.51 & 49.43 & 36.15 \\
\hline & Std. error & 0.82 & 0.46 & 3.07 & 2.68 \\
\hline & Std. dev. & 3.75 & 2.11 & 17.08 & 12.26 \\
\hline \multirow{5}{*}{ Ra nano., nm } & Minimum & 4.98 & 6.76 & 7.98 & 7.56 \\
\hline & Maximum & 8.78 & 9.88 & 22.57 & 12.66 \\
\hline & Arithm. mean & 6.54 & 8.22 & 11.31 & 9.51 \\
\hline & Std. error & 0.19 & 0.22 & 1.02 & 0.29 \\
\hline & Std. dev. & 0.87 & 1.02 & 4.68 & 1.35 \\
\hline \multirow{5}{*}{ HAP size, nm } & Minimum & 28 & 48 & 33 & 35 \\
\hline & Maximum & 49 & 74 & 81 & 80 \\
\hline & Arithm. mean & 38.38 & 62 & 54.05 & 53.24 \\
\hline & Std. error & 1.31 & 1.92 & 3.61 & 3.72 \\
\hline & Std. dev. & 6 & 8.82 & 16.53 & 17.05 \\
\hline
\end{tabular}
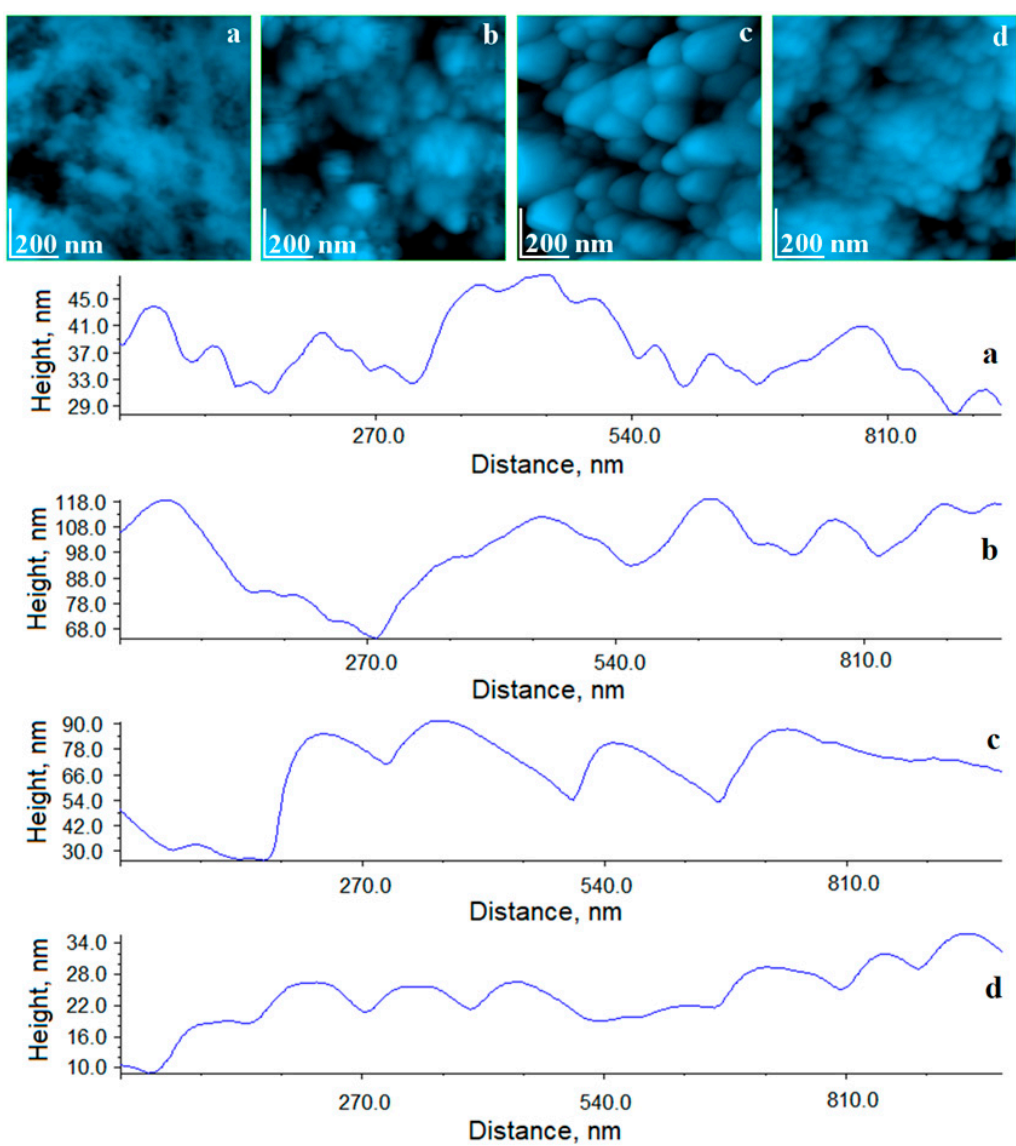

Figure 12. AFM topographic images at the nanostructure of the samples of (a) healthy, untreated enamel, (b) coffee stained enamel, (c) stained enamel and treated with Opalescence gel, and (d) stained enamel and treated with experimental gel. Two-dimensional profiles on the horizontal median of the topographic images are given. They are related to the roughness at the nanostructure level, "Ra nano", in Table 2. 


\section{Discussion}

The results of the study indicate more important antimicrobial activity in the case of inoculation of 50 , as well as $100 \mu \mathrm{l}$ extract solutions of $50 \%$ concentration. The most significant antimicrobial activity was present in the case of Peptostreptococcus anaerobius. Some studies have shown the presence of P. anaerobius in almost $90 \%$ of cases of periodontal disease in both women and men [17]. The pathogens of periodontal disease have been detected not only in sick patients, but also in those who are apparently healthy [17-19]. Research has also shown the presence of P. anaerobius in root reinfections resulting from failed endodontic treatments [20-22].

On the Streptococcus mutans strain, the extract solution showed significant antimicrobial activity, while the response of the $C$. xerosis strain was sensitive, depending on the amount and concentration of the experimental extract. On the C. albicans strain, the experimental formula based on Camellia sinensis had no antifungal effect.

Streptococcus mutans is a bacteria commonly found in the oral cavity. With the help of glycosyl transferase, the bacteria produce insoluble glucans, which adhere to the tooth surface, starting the formation of dental plaque. Under this film, the proliferation of Streptococcus mutans occurs. During the proliferation process, sucrose produces lactic acid, which contributes to the demineralization of dental hard tissues [23]. It has also been shown that the active substances contained in green tea extract, such as catechins, have the ability to inhibit the secretion of glucan, a substance secreted by Streptococcus mutans [24,25]. This substance is involved in the formation of bacterial plaque [26,27]. Thus, both by inhibiting the growth of Streptococcus mutans, the main cariogenic bacteria, and by inhibiting the proliferation of bacterial plaque, we can say that green tea extract has a carioprotective effect. In vivo and in vitro studies also support the anticariogenic effect of green tea extract by inhibiting the attachment of Streptococcus mutans to hydroxyapatite crystals [28].

Candida albicans is a saprophytic bacterial species, located in the oral and vaginal mucosa [29], capable of causing infections in the case of decreased immune defense of the host organism, such as immunosuppressive infections, antibiotic medication, and immaturity of the immune system (newborns). Some studies have shown that low $\mathrm{pH}$ levels encourage the production and expansion of Candida albicans colonies. It is also well known that a low $\mathrm{pH}$ in the oral cavity allows the development of dental plaque, especially in patients with high sugar diet [30,31]. It has been shown that in the presence of Candida albicans, the production of glucans secreted by Streptococcus mutans is increased and also the destructive capacity of Streptococcus mutans is increased [32]. In 1991, in the first study on the effect of green tea on Candida albicans, the result was negative [33]. It has been shown that low levels of EGCG, the main active component of green tea extract, have no antifungal action [34]. Our results did not show any anti-fungal activity of the experimental extract. Previous studies have shown the antifungal effect of green tea only after it reached a $\mathrm{pH}$ of $6.5[35,36]$. Recent research in sensing technology concluded that $\mathrm{pH}$ sensors could be used in vivo or in vitro studies, due to their properties, such as biocompatibility, high precision, and fast and stable responses, in order to better understand the chemical processes during therapeutic and esthetic procedures in dentistry [37,38]. Other research has found that the most effective antifungal action is the combination of green tea extract and chloramphenicol [36].

Numerous researches have shown that the effects of bleaching substances on tooth enamel are directly related to the concentration of carbamide peroxide; the higher the concentration, the more the surface roughness of the enamel suffers [39]. This process is attributed to the oxidative action of the bleaching agent on the organic phase of the dental hard tissues [40]. Other studies have shown that increasing the roughness of enamel after bleaching can promote the adhesion of Streptococcus mutans to the tooth surface [41].

Saliva has a cleansing function, but it also has a buffering action due to bicarbonate and phosphate systems. Some inorganic electrolytes contained in saliva (calcium, phosphorus, and fluoride) are important participants in the remineralization process. When the $\mathrm{pH}$ is below the physiological limit, some of the calcium and phosphorus complexes are released and added to the ionic-calcium 
and phosphorus reservoirs. Consequently, the apatite on the enamel surface is protected against dissolution [42,43].

Gonzalez-Lopez et al. reported that bleaching changed the organic composition in the enamel and decreased the phosphate and carbonate content, thus making the enamel surface rough and uneven $[43,44]$.

In this context, researchers' attention turned to the study of the actions of vegetable decoctions, which have been used since ancient times in both traditional Eastern and Western medicine for their anti-inflammatory and antibacterial effects [36]. Recently, plants with healing properties have come to symbolize safety, as opposed to drugs made by chemical synthesis, which are now considered unsafe for both patients and the environment [45]. Increasing the use of medicinal plants in medicine offers an important economic advantage in treating various diseases [46]. Numerous natural extracts have been researched, such as propolis, orange oil, chamomile, and green tea. In this context, previous studies have concluded that green tea is a good chelating agent [47] and inhibits the enzyme gyrase by binding to ATP (adenosine triphosphate) [48]. Some research has shown a good antibacterial effect of green tea on endodontic microflora, including E. faecalis [49]. In periodontics, the literature has shown that green tea has the ability to inhibit lipopolysaccharide, which induces bone resorption [50]. It has also been shown that the active substances in green tea extract, as well as in catechism, are a good adjunct during the professional hygiene procedure, by descaling and root planing of patients with chronic periodontitis [51]. On cariogenic bacteria, some studies have shown the positive actions of green tea by inhibiting the growth of $S$. mutans by decreasing bacterial adhesion on the tooth surface [51].

Some studies have concluded that before the application of remineralizing agents, the application of tea-based preparations may be an acceptable option to remineralize demineralized dental hard tissues [52,53]. Other researchers concluded in a study that in addition to fluoride, the organic components of tea also have the property of increasing the acid resistance of tooth enamel. It also suggests that organic components appear to play a key role in their actions, rather than fluoride [54]. Another study showed that toothpaste containing $15 \%$ green tea extract favored the remineralization of bleached enamel, showing an increased value of micro roughness [55].

\section{Conclusions}

The green tea extract solution exerts an important antibacterial effect on P. anaerobius and S. mutans strains, the values of the inhibition diameters having values close to those of the reference antibiotics recommended by CLSI.

The natural extract based on Camellia sinensis has antimicrobial activity, conditioned by the dose against $C$. xerosis. The experimental extract has no antifungal action on the $C$. albicans strain. At the end of the bleaching treatment, both experimental groups showed a modified structure compared to the untreated control group.

Following Opalescence gel whitening, the surface of the enamel has a modified architecture compared to that of the healthy, untreated enamel, and the roughness has higher values than the control group.

The experimental group bleached with gel based on green tea extract and carbamide peroxide, although showing a prismatic appearance similar to that of the group bleached with Opalescence, had a roughness close to that of the control group, untreated due to the protective film of the green tea extract.

Author Contributions: Conceptualization, A.M. and A.M. Investigation, A.D., I.P., R.E., and S.P.; Methodology, V.P. and M.M.; Software, M.V.; Supervision, A.M. and V.P.; Writing-original draft, C.V. All authors have read and agreed to the published version of the manuscript.

Funding: This research received no external funding.

Conflicts of Interest: The authors declare no conflict of interest. 


\section{References}

1. Lazarescu, F. Incursiune în estetica dentară; SSER Publishing House: Bucharest, Romania, 2013.

2. Forester, S.C.; Lambert, J.D. Antioxidant effects of green tea. Mol. Nutr. Food Res. 2011, 55, $844-854$. [CrossRef] [PubMed]

3. Senanayake, S.P.J.N. Green tea extract: Chemistry, antioxidant properties and food applications-A review. J. Funct. Foods. 2013, 5, 1529-1541. [CrossRef]

4. Giangreco, F.; Höfinger, S.; Bakalis, E.; Zerbetto, F. Impact of the green tea ingredient epigallocatechin gallate and a short pentapeptide (Ile-Ile-Ala-Glu-Lys) on the structural organization of mixed micelles and the related uptake cholesterol. Biochim. Biophys. Acta Gen. Subj. 2018, 9, 1956-1963. [CrossRef] [PubMed]

5. Morin, M.P.; Grenier, D. Regulation of matrix mettaloproteinase secretion by green tea catechins in a three-dimendional co-culture model of macrophages and gingival fibroblasts. Arch. Oral Biol. 2017, 75, 89-99. [CrossRef] [PubMed]

6. Ramenzanali, F.; Samimi, S.; Kharazifard, M.; Afkhami, F. The in vitro antibacterial afficacy of Persian green tea extract as an intracanal irrigant on Enterococcus faecalis biofilm. Iran Endod. J. 2016, 11, 304-308.

7. Stuart, C.H.; Schwartz, S.A.; Beeson, T.J.; Owatz, C.B. Enterococcus faecalis: its role in root canal treatment failure and current concepts in retreatment. J. Endod. 2006, 32, 93-98. [CrossRef]

8. Gomes, B.P.; Pinheiro, E.T.; Jacinto, R.C.; Zaia, A.A.; Terraz, C.C.; Souza-Filho, F.J. Microbial analysis of canals of root filled teeth with periapical lesions using polymerase chain reaction. J. Endod. 2008, 34, 537-540. [CrossRef]

9. Agrawal, V.; Kapoor, S.; Agrawal, I. Critical review on eliminating endodontic dental infections using herbal products. J. Diet. Suppl. 2017, 14, 229-240. [CrossRef]

10. Silva, B.G.; Gouveia, T.H.; da Silva, M.D.; Ambrosano, G.M.; Aguiar, F.H.; Lima, D.A. Evaluation of home bleaching gel modified by different thickeners on the physical properties of enamel: An in situ study. Eur. J. Dent. 2018, 12, 523-527. [CrossRef]

11. de Oliveira, R.P.; Baia, J.C.; Ribeiro, M.E.; e Souza, M.H. Influence of time intervals between bleaching procedures on enamel microhardness and surface roughness. Open. Dent. J. 2018, 12, 555. [CrossRef]

12. González-Ochoa, J.G. Histological changes to dental pulp after vital bleaching with $10 \%$ carbamide peroxide. Master's Thesis, Indiana University School of Dentistry, Indianapolis, IN, USA, 2002.

13. de Abreu, D.R.; Sasaki, R.T.; Amaral, F.L.; Florio, F.M.; Basting, R.T. Effect of home-use and in-office bleaching agents containing hydrogen peroxide associated with amorphous calcium phosphate on enamel microhardness and surface roughness. J. Esthet. Restor. Dent. 2011, 23, 158-168. [CrossRef] [PubMed]

14. Tam, L. The safety of home bleaching techniques. J. Can. Dent. Assoc. 1999, 65, 453-455. [PubMed]

15. Lombardini, M.; Ceci, M.; Colombo, M.; Bianchi, S.; Poggio, C. Preventive effect of different toothpastes on enamel erosion: AFM and SEM studies. Scanning 2014, 36, 401-410. [CrossRef] [PubMed]

16. Cerci, B.B.; Roman, L.S.; Guariza-Filho, O.; Camargo, E.S.; Tanaka, O.M. Dental enamel roughness with different acid etching times: Atomic force microscopy study. Eur. J. Gen. Dent. 2012, 3, 187-191.

17. Higaki, S.; Kitagawa, T.; Kagoura, M.; Morohashi, M.; Yamagishi, T. Characterization of Peptostreptococcus species in skin infections. Int. J. Med. Res. 2000, 28, 143. [CrossRef]

18. Boskey, E.R.; Telsch, K.M.; Whaley, K.J.; Moench, T.R.; Cone, R.A. Acid production by vaginal flora in vitro is consistent with the rate and extent of vaginal acidification. Infect. Immun. 1999, 67, 5170. [CrossRef]

19. Moore, W.E.; Holdeman, L.V.; Cato, E.P.; Smibert, R.M.; Burmeister, J.A.; Ranney, R.R. Bacteriology of moderate (chronic) periodontitis in mature adult humans. Infect. Immun. 1983, 42, 510. [CrossRef]

20. Heeyoung, C.; Eunhye, K.; Jihoon, K.; Hyun-Joo, K.; Yo-Youn, L.; Yeonul, C.; Ji-Young, J. Real time PCR quantifi-cation of 9 periodontal pathogens in saliva samples from periodontally healthy Koreean young adults. J. Periodontal Implant. Sci. 2018, 48, 261.

21. Tonea, A.; Oana, L.; Badea, M.; Sava, S.; Voina, C.; Ranga, F.; Vodnar, D. Hplc analysis, antimicrobial and antifungal activity of an experimental plant based gel, for endodontic usage. Studia Univ. Babes-Bolyai Chem. 2016, 61, 53.

22. Rôças, I.N.; Siqueira, J.F. Root canal microbiota of teeth with chronic apical periodontitis. J. Clin. Microbiol. 2008, 46, 3599. [CrossRef]

23. Munson, M.A.; Pitt-Ford, T.; Chong, B.; Weightman, A.; Wade, W.G. Molecular and cultural analysis of the microflora associated with endodontic infections. J. Dent. Res. 2002, 81, 761-766. [CrossRef] 
24. Sundqiust, G. Associations between microbial species in dental root canal infections. Oral Microbiol. Immunol. 1992, 7, 257-262. [CrossRef]

25. Dhariwal, N.S.; Hugar, S.M.; Harakuni, S.; Sogi, S.; Assudani, H.G.; Mistry, L.N. A comparative evaluation of antibacterial effectiveness of sodium hypochlorite, Curcuma longa, and Camellia sinensis as irrigating solutions on isolated anaerobic bacteria from infected primary teeth. J. Indian Soc. Pedod. Prev. Dent. 2016, 34, 165-171. [CrossRef]

26. Lipsky, B.A.; Goldberger, A.C.; Tompkins, L.S.; Plorde, J.J. Infections caused by nondiphtheria corynebacteria. Rev. Infect. Dis. 1982, 4, 1220-1235. [CrossRef]

27. Khemaleelakul, S.; Baumgartner, J.C.; Pruksakorn, S. Identification of bacteria in acute endodontic infections and their antimicrobial susceptibility. Oral Surg. Oral Med. Oral Pathol. Oral Radiol. Endod. 2002, 94, 746-755. [CrossRef]

28. Zaidi, M.; Tayyab, K.; Wajahat, A.; Shafique, M.; Naz, S.A. Inhibitory effect of natural herbal extracts on systemic bacteria. Int. J. Biol. Res. 2013, 1, 89-93.

29. Sakagami, H.; Watanabe, T.; Hoshino, T.; Suda, N.; Mori, K.; Yasui, T.; Yamauchi, N.; Kashiwagi, H.; Gomi, T.; Oizumi, T.; et al. Recent progress of basic studies of natural products and their dental application. Medicines 2019, 6, 4. [CrossRef]

30. Karygianni, L.; Al-Ahmad, A.; Argyropoulou, A.; Hellwig, E.; Anderson, A.C.; Skaltsounis, A.L. Natural antimicrobials and oral microorganisms:a systematic review on herbal interventions for eradication of multispecies oral biofilms. Front. Microbiol. 2015, 6, 1529.

31. Ooshima, T.; Minami, T.; Aono, W.; Izumitani, A.; Sobue, S.; Fujiwara, T.; Kabawata, S.; Hamada, S. Oolong tea polyphenols inhibit experimental dental caries in SPF rats infected with mutans streptococci. Caries Res. 1993, 27, 124-129. [CrossRef]

32. Nakahara, K.; Kawabata, S.; Ono, H.; Tanaka, T.; Ooshima, T.; Hamada, S. Inhibitory effect of oolong tea polyphenols of glycosyltransferases of mutans streptococci. Appl. Environ. Microbiol. 1993, 59, 968-973. [CrossRef]

33. Otake, S.; Makimura, M.; Kuroki, T.; Nishihara, Y.; Hirasawa, M. Anticaries effects of polyphenolic compounds from Japanese green tea. Caries Res. 1991, 25, 438-443. [CrossRef]

34. Rasheed, A.; Haider, M. Antibacterial activity of Camellia sinensis extracts against dental caries. Arch. Pharm. Res. 1998, 21, 348-352. [CrossRef]

35. Al-Talib, H.; Kasim, N.A.; Al-Khateeb, A.; Murugaiah, C.; Abdul, A.; Aziz, N.N.; Azizan, N.; Ridzuan, S. Antimicrobial effect of malaysian green tea leaves (camellia sinensis) on the skin microbiota. Malays. J. Microbiol. 2016, 12, 423-427.

36. Gordon, N.C.; Wareham, D.W. Antimicrobial activity of the green tea polyphenol (-)-epigallocatechin-3-gallate (EGCG) against clinical isolates of Stenotrophomonas maltophilia. Int. J. Antimicrob. Agents. 2010, 36, 129-131. [CrossRef]

37. Pathak, A.K.; Bhardwaj, V.; Gangwar, R.K.; De, M.; Singh, V.K. Fabrication and characterization of $\mathrm{TiO}_{2}$ coated cone shaped nano-fiber $\mathrm{pH}$ sensor. Opt. Commun. 2017, 386, 43-48. [CrossRef]

38. Aldaba, A.L.; González-Vila, Á.; Debliquy, M.; Lopez-Amo, M.; Caucheteur, C.; Lahem, D. Polyaniline-coated tilted fiber Bragg gratings for $\mathrm{pH}$ sensing. Sens Actuators B Chem. 2018, 254, 1087-1093. [CrossRef]

39. Eimar, H.; Siciliano, R.; Abdallah, M.N.; Nader, S.A.; Amin, W.M.; Martinez, P.P.; Celemin, A.; Cerruti, M.; Tamimi, F. Hydrogen peroxide whitens teeth by oxidizing the organic structure. J. Dent. 2012, 40, 25. [CrossRef]

40. Nam, S.H.; Ok, S.M.; Kim, G.C. Tooth bleaching with low-temperature plasma lowers surface roughness and streptococcus mutans adhesion. Int. Endod. J. 2018, 51, 479-488. [CrossRef]

41. Justino, L.M.; Tames, D.R.; Demarco, F.F. In situ and in vitro effects of bleaching with carbamide peroxide on human enamel. Oper. Dent. 2004, 29, 219-225.

42. Thylstrup, A.; Fejerskov, O. Características clínicas e patológicas da cárie dentária. In THYLSTRUP, A.; FEJERSKOV, O. Cariologia Clínica, 3rd ed.; Livraria Editora Santos: São Paulo, Brazil, 1995; Volume 2, pp. 111-157.

43. González-López, S.; Torres-Rodríguez, C.; Bolanos-Carmona, V.; Sanchez-Sanchez, P.; Rodríguez-Navarro, A.; Alvarez-Lloret, P.; Garcia, M.D. Effect of 30\% hydrogen peroxide on mineral chemical composition and surface morphology of bovine enamel. Odontology. 2016, 104, 44. [CrossRef] 
44. Zhang, H.; Shao, S.; Du, A.; Wang, Y.; Cheng, B.; Zhang, Z. Comparative evaluation of two antioxidants on reversing the immediate bond strength of bleached enamel: In vitro study. Med. Sci. Monit. 2020, 26, e920481. [CrossRef] [PubMed]

45. Archana, S.; Abraham, J. Comparative analysis of antimicrobial activity of leaf extracts from fresh green tea, commercial green tea and black tea on pathogens. J. Appl. Pharm. Sci. 2011, 8, 149.

46. Hirasawa, M.; Takada, K. Multiple effects of green tea catechin on the antifungal activity of antimycotics against Candida albicans. J. Antimicrob. Chemother. 2004, 53, 225-229. [CrossRef]

47. Verran, J. Preliminary studies on denture plaque microbiologyand acidogenicity. Microbiol. Ecol. Health Dis. 1988, 1, 51-55. [CrossRef]

48. Samaranayake, L.P. Nutritional factors and oral candidosis. J. Oral. Pathol. 1986, 15, 61-65. [CrossRef]

49. Falsetta, M.L.; Klein, M.I.; Colonne, P.M.; Scott-Anne, K.; Gregoire, S.; Pai, C.H.; Gonzalez-Begne, M.; Watson, G.; Krysan, D.J.; Bowen, W.H.; et al. Symbiotic relationship between Streptococcus mutans and Candida albicans synergizes virulence of plaque biofilms in vivo. Infect. Immun. 2014, 82, 1968-1981. [CrossRef]

50. Nakamura, H.; Ukai, T.; Yoshimura, A.; Kozuka, Y.; Yoshioka, H.; Yoshinaga, Y.; Abe, Y.; Hara, Y. Green tea catechin inhibits lipopolysaccharide-induced bone resorption in vivo. J. Periodontal Res. 2010, 45, 23. [CrossRef]

51. Steinmann, J.; Buer, J.; Pietschmann, T.; Steinmann, E. Anti-infective properties of epigallocatechin-3-gallate (EGCG), a component of green tea. Br. J. Pharmacol. 2013, 168, 1059-1070. [CrossRef]

52. Cvikl, B.; Lussi, A.; Moritz, A.; Flury, S. Enamel surface changes after exposure to bleaching gels containing carbamide peroxide or hydrogen peroxide. Oper. Dent. 2016, 41, 39-47. [CrossRef]

53. Khoroushi, M.; Shirban, F.; Doustfateme, S.; Kaveh, S. Effect of three nanobiomaterials on the surface roughness of bleached enamel. Contemp. Clin. Dent. 2015, 6, 466-470.

54. Mazilu, A.; Sarosi, C.; Moldovan, M.; Filip, M.; Prodan, D.; Antoniac, A.; Prejmerean, C.; Silaghi-Dumitrescu, L.; Popescu, V.; Raiciu, A.D.; et al. Preparation and characterization of natural bleaching gels used in cosmetic dentistry. Materials 2019, 12, 2106. [CrossRef] [PubMed]

55. Dionysopoulos, D.; Koliniotou-Koumpia, E.; Tolidis, K.; Gerasimou, P. Effect of fluoride treatments on bleached enamel microhardness and surface morphology. Oral Health. Prev. Dent. 2017, 15, 169-175. 